\title{
DEL APRENDIZAJE SIGNIFICATIVO AL APRENDIZAJE SUSTENTABLE. PARTE 2: DERIVACIONES COMUNICACIONALES Y DIDÁCTICAS
}

\section{Galagovsky, Lydia R.}

Centro de Formación e Investigación en Enseñanza de las Ciencias Facultad de Ciencias Exactas y Naturales. Universidad de Buenos Aires Ciudad Universitaria. Pabellón II. 1428 Buenos Aires. Argentina lyrgala@qo.fcen.uba.ar

Resumen. El aula es un espacio de comunicación entre la mente del docente (experto) y las mentes de los alumnos (novatos). En este contexto, el modelo de aprendizaje cognitivo consciente (MACCS) permite la reinterpretación y resignificación tanto de procedimientos de enseñanza como de procesos de aprendizaje.

En el presente trabajo, se analizan aspectos semánticos y sintácticos de los lenguajes como obstáculos para las habilidades comuncacionales en el aula. Asimismo, cuestionamos, desde fundamentos epistemológicos, acciones didácticas tradicionales como las de indagar ideas previas, generar conflictos cognitivos y promover el cambio conceptual, proponiendo alternativas fundamentadas en el MACCS.

Palabras clave. Modelo, aprendizaje sustentable, conflicto cognitivo, ideas previas, cambio conceptual.

Summary. Communication between the teacher's (expert) and the students' (novices) minds takes place in the classroom. In this context, we propose a Sustainable Cognitive Model of Conscious Learning (SCMCL) that permits reinterpretations and redefinitions both of teaching procedures and of learning processes.

In this paper, we analyze syntactic and semantic aspects of languages as obstacles for communicational skills in the classroom. We also dispute, from an epistemological base, traditional teaching activities such as finding out preconceptions, creating cognitive conflicts, and promoting conceptual changes, putting forth alternatives based on SCMCL.

Keywords. Model, sustainable learning, cognitive conflict, preconceptions, conceptual change.

\section{INTRODUCCIÓN}

La propuesta teórica de un modelo de aprendizaje cognitivo consciente sustentable (MACCS), presentada en la Parte 1 (Galagovsky, 2004) describe en forma sencilla, mediante lenguajes verbal y gráfico, complejos procesos de aprendizaje. La figura 1 recrea gráficamente los puntos sobresalientes del MACCS, que son los que se exponen a continuación.

\section{Diferenciación entre información y conocimiento}

La palabra conocimiento remite a lo que sabe un sujeto, a lo que tiene en su mente. Nuestra propuesta llama información al bagaje de datos, conceptos y relaciones, que están fuera de las mentes de los sujetos; es decir, información es el conjunto o las partes de todos los saberes 
documentados (impresos, vídeo, internet, etc.) que una sociedad o una civilización ha desarrollado sobre determinados temas (Fig. 1a).

Durante el aprendizaje, la información (externa al sujeto) se transforma en conocimiento (existente en la mente del sujeto).

\section{Diferenciación entre aprendizaje sustentable y apren- dizaje aislado}

El aprendizaje sustentable (Fig. 1b) es aquél en el que la información recibida -o parte de ella- fue apropiada correctamente como nuevo conocimiento, aumentando y enriqueciendo la estructura cognitiva previamente existente. Los conceptos que han funcionado como nexos correctos se denominan conceptos sostén; en la figura $1 \mathrm{~b}$ son los dos que aparecen sombreados. Como resultado de este tipo de aprendizaje, la estructura cognitiva del sujeto se enriquecerá con conocimiento sustentable, y sus conceptos podrán actuar como nuevos conceptos sostén.

El aprendizaje aislado (Fig. 1c) aparece cuando un sujeto no logra con éxito vincular a conocimientos previos una información externa que recibe. Para retener esta información como conocimiento deberá usar estrategias de memorización y su mente guardará conocimiento exclusivamente memorístico y aislado.

Figura 1

Tipos de aprendizaje definidos para el modelo de aprendizaje cognitivo consciente sustentable (MACCS)

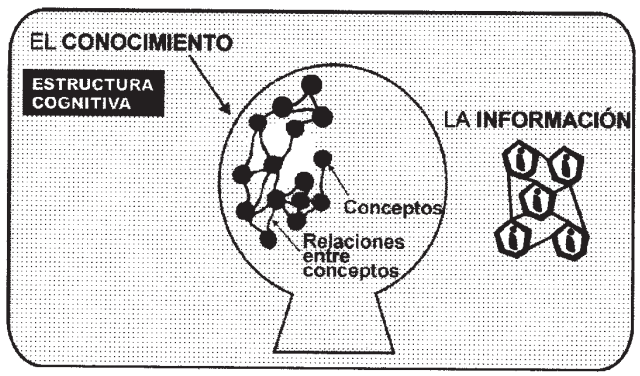

Figura 1a: Diferencias entre información y conocimiento El conocimiento es una intrincada red de conceptos y relaciones entre conceptos que están dentro de la cabeza de un sujeto. La información está afuera de la cabeza de los sujetos.

Figura 1b: Aprendizaje sustentable

Parte de la información externa es procesada y consolidada como nuevo conocimiento -círculos con la letra $i-$, vinculándose al ya existente. Los conceptos sostén-sombreados en el dibujo- son el nexo de vinculación.

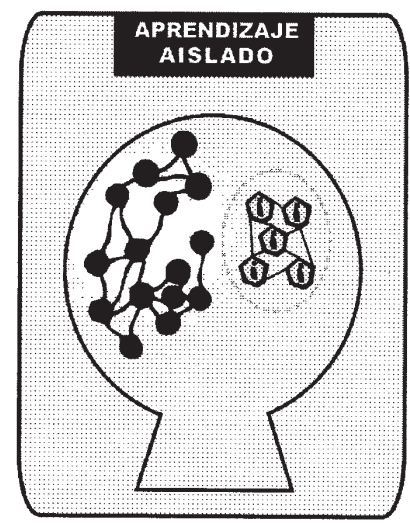

Figura 1c: Aprendizaje aislado

La información externa no es vinculada al conocimiento existente.

Este tipo de aprendizaje puede evocarse y explicitarse de tal forma que un evaluador externo podría no darse cuenta de esta condición de aislamiento conceptual. 


\section{Diferenciación entre conceptos inclusores y concep- tos sostén}

Novak $(1984,1999)$ ha otorgado a los conceptos inclusores una función de nexo entre el nuevo y el viejo aprendizaje. En nuestro análisis previo (Parte 1 del presente trabajo, Galagovsky, 2004), hemos discutido la posibilidad de que un concepto inclusor sea incorrecto; en este caso, podrán originarse aprendizajes significativos erróneos ${ }^{1}$, muy resistentes a cualquier cambio conceptual. El MACCS establece que los conceptos sostén deben ser los conceptos nexo científicamente correctos.

\section{Restricción a procesos cognitivos conscientes}

Tanto en la descripción verbal como en las representaciones gráficas, el MACCS sólo hace referencia a la parte cognitiva consciente de la estructura de la mente. La estructura afectiva, emotiva o vincular, existe y es la fuerza vital de cada sujeto; su importancia es sustancial a la hora de predisponerlo para el aprendizaje consciente, pero sus complejas variables no están representadas en el MACCS.

El presente trabajo indica cómo el MACCS aporta el andamiaje teórico para entender qué mecanismos preparatorios de la mente de los alumnos podrán optimizarse antes de recibir éstos la información científica a aprender. Los rasgos sencillos del MACCS tienen una fuertísima implicancia didáctica porque contienen elementos teóricos que fuerzan a resignificar, a reconsiderar conceptualmente y a redefinir operacionalmente acciones didácticas como las de indagación de ideas previas, generación de conflicto cognitivo y necesidad de lograr un cambio conceptual en los alumnos.

El primer punto del MACCS (Fig. 1a), señala un punto esencial como primera traba para lograr un aprendizje sustentable: la información, externa al sujeto -proveniente del discurso del docente, de libros, etc.- debe poder ser procesada por las capacidades congitivas del sujeto que va a aprender. Se está frente a un problema de comunicación que involucra diferentes aspectos detallados a continuación.

\section{DERIVACIONES COMUNICACIONALES DEL MACCS}

La clase es un lugar de intercambio comunicacional entre un docente -un experto- y sus alumnos. Los lenguajes constituyen los canales de esa comunicación. Un aspecto básico para lograr una buena comunicación es que los mensajes emitidos sean decodificables por el receptor del mensaje. Para decodificar se requiere que emisor y receptor compartan códigos y significaciones; es decir, que acuerden en sintaxis y semántica.

Una óptima calidad de comunicación se establecerá en el aula cuando alumnos entre sí y con el docente puedan compartir el significado de palabras, oraciones, gráficos con sus códigos y formatos sintácticos (Rodríguez, 2001; Seferián, 2002). Brechas entre sendas significaciones debidas a diferencias de complejidad entre los saberes del docente experto y de sus alumnos ocasionan obstáculos epistemológicos infranqueables para el aprendizaje sustentable.

\subsection{Los lenguajes}

En un trabajo anterior (Galagovsky et al., 2003) hemos propuesto una clasificación posible de tipos de lenguajes utilizados complementariamente por un experto en química. La figura 2 extiende dicha clasificación a un docente de alguna de las ciencias naturales. En esta clasificación, consideramos que el nivel macroscópico es el referido a los eventos percibidos por los sentidos: un vaso lleno con un líquido incoloro y burbujas que suben puede ser igualmente percibidos por el docente y sus alumnos. Sin embargo, decir que «es agua en ebullición» o que «son burbujas de $\mathrm{CO}_{2}$ debidas al agregado de un ácido a una solución de bicarbonato» son explicaciones en lenguaje verbal, originadas en interpretaciones del fenómeno, claramente ubicadas en el nivel simbólico.

En la figura 2 mantenemos el lenguaje visual separado del resto para señalarlo como aquel medio perceptivo por el cual recibimos las señales provenientes de hechos macroscópicos, sin interpretación simbólica alguna. Un vídeo sobre la interacción de ciertas moléculas, aunque claramente debe ser percibido a través del sentido de la vista, lo ubicamos como un lenguaje gráfico, ya que describe dinámicamente un contenido simbólico. Es decir, define si el lenguaje es visual, o de algún otro tipo, no que esté basado en imágenes, sino cuál es el contenido referencial de esas imágenes.

Decimos en la figura 2 que los diferentes lenguajes utilizan códigos y formatos sintácticos. Los códigos se refieren a convenciones normativas -establecidas por consenso por los expertos en una disciplina- que dan validez a los elementos que conforman los lenguajes. Los formatos sintácticos -también basados en convenciones- son elecciones sobre las caracterísiticas de las unidades semánticas aceptadas para expresar los contenidos de dicha disciplina. Estos conceptos se amplían a continuación.

\subsection{Los códigos}

En la figura $3 a$ y $3 b$ se muestran dos formas de representar la molécula de agua en lenguaje gráfico (Fig. 2), mediante las clásicas esferitas. Estos dibujos simples tienen implícitos gran número de códigos que encapsulan conocimientos que un experto maneja con soltura:

- Se dibujan esferitas «sólidas»; sin embargo, el volumen de los átomos no se corresponde con su distribución de masa.

- El color de cada esfera es arbitrario. 
Figura 2

Niveles de interpretación y lenguajes utilizados complementariamente por un experto en alguna disciplina científica.

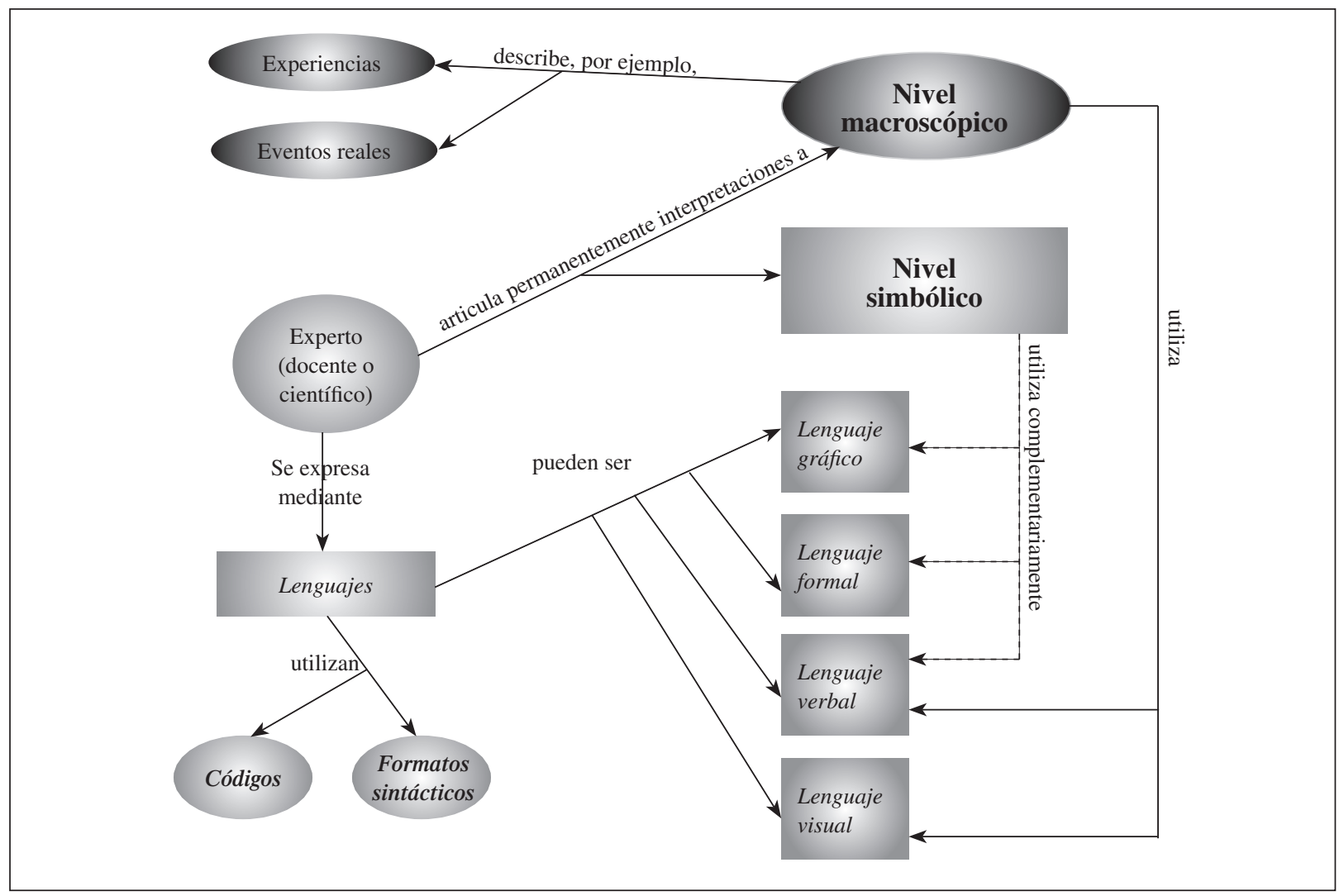

- El ángulo de $105^{\circ}$ se explica mediante un modelo de hibridización del oxígeno. Un experto evaluaría como incorrecto un dibujo de la molécula de agua donde los átomos se ubicaran linealmente. Su importancia radica en que este ángulo determina la existencia de un momento dipolar en la molécula que tiene consecuencias sobre el estado de agregación del agua a temperatura ambiente -el agua es un líquido y no un gas- y sobre su menor densidad en el estado sólido que en el estado líquido.

En la figura 3c, se utilizan fórmulas que involucran códigos diferentes a los anteriores: desde nuestro punto de vista, la utilización de fórmulas químicas implica la utilización de un lenguaje formal (Fig. 2).

Los códigos podrían enunciarse de la siguiente manera:

- Se utilizan las letras que designan los elementos químicos como si fueran los núcleos de cada uno de ellos y la totalidad de sus electrones, excepto los electrones de valencia.

- Se utilizan segmentos como nexo entre letras para representar las uniones químicas no iónicas entre los átomos; es decir, así se manifiesta una vinculación estable de electrones en un orbital molecular ocupado.

La figura 3d también utiliza lenguaje formal, pero esta vez el código intenta mostrar que el ángulo y la formación de puentes de hidrógeno determinan la particularidad de que, en el hielo, la densidad sea menor que en el agua líquida, así como que la sustancia agua sea líquida y no gaseosa a una atmósfera de presión y temperatura ambiente.

\subsection{Los formatos sintácticos}

Todo lenguaje tiene una forma apropiada y típica de ordenar la secuencia de sus elementos sintácticos.

En la figura 3e se muestra un formato sintáctico apropiado y dos incorrectos para describir la síntesis química de agua a partir de sus elementos constituyentes. Todas las ecuaciones están balanceadas, pero en la segunda se repite, en la zona de reactivos y de productos, una misma sustancia (el oxígeno), y ésta no es una sintaxis aceptada para el lenguaje formal que expresa reacciones químicas. Por otra parte, la sintaxis apropiada exige una flecha con punta entera apuntando hacia la derecha, que es el código aceptado para significar una reacción completa, aunque no indica espontaneidad en condiciones normales de presión y temperatura. La tercera ecuación muestra una doble semiflecha; este código indica reversibilidad de la reacción, y eso es químicamente incorrecto para el caso de la obtención del agua. Por lo tanto, la sintaxis de esta ecuación es incorrecta. 
Figura 3

Códigos y formatos sintácticos para el concepto de agua.

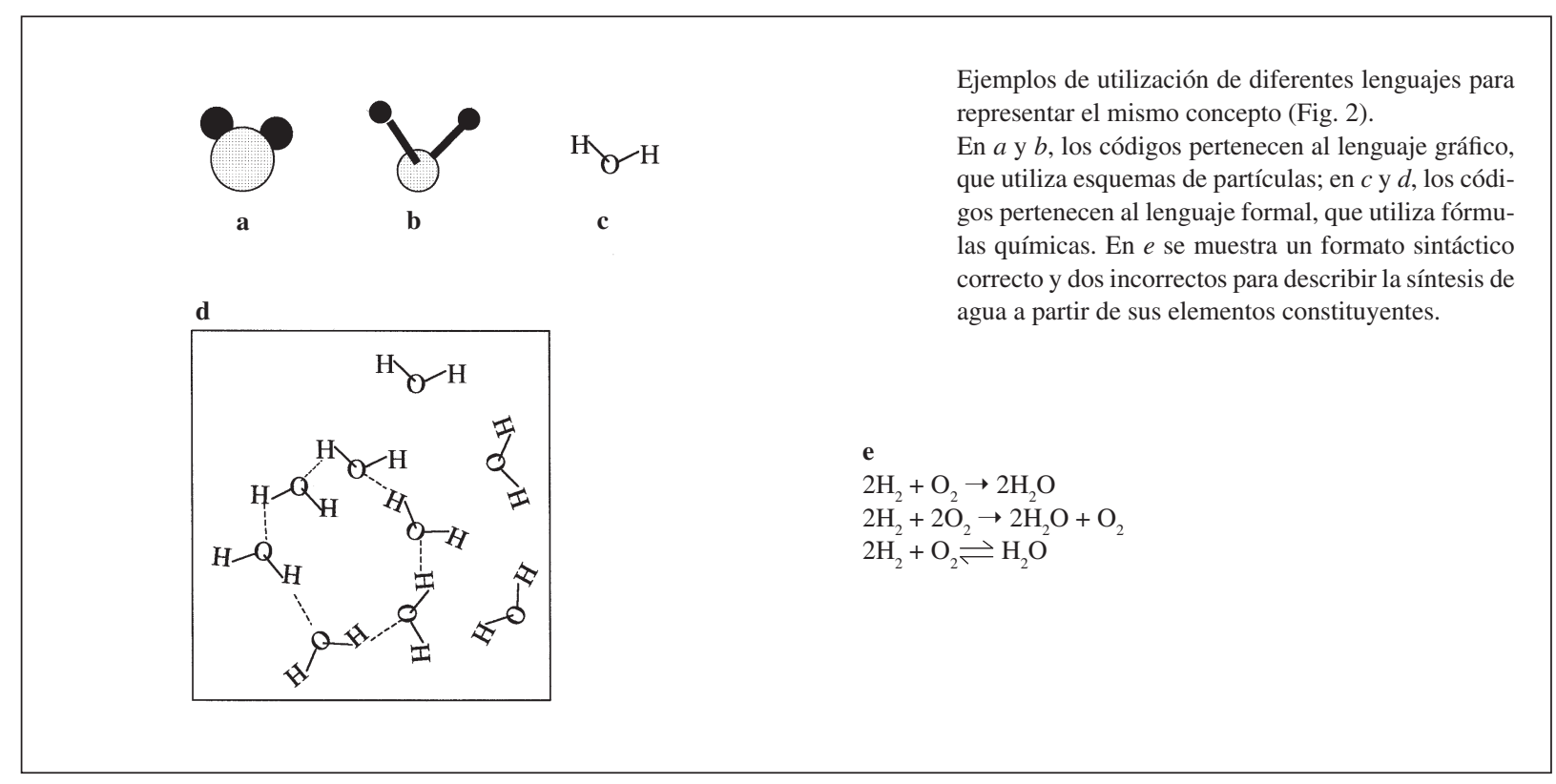

Todas estas consideraciones nos llevan a confirmar que los expertos utilizamos una variedad de lenguajes que encapsulan gran cantidad de información en sus códigos y formatos sintácticos, tal como describimos en la figura 2 .

\subsection{Los modos de representación (MDR) de J. Fodor}

Jerry Fodor, en su ensayo sobre si «el significado es información» (1998, pp. 30 y 34), discute el uso de diferentes lenguajes introduciendo el término modos de representación $(\mathrm{MDR})$ :

«Y supóngase que ser agua y ser $\mathrm{H}_{2} \mathrm{O}$ son la misma propiedad $^{2}$. Entonces, se sigue que, si es una ley que las instancias de AGUA covarían con agua, también es una ley que las instancias de AGUA covarían con $\mathrm{H}_{2} \mathrm{O}$. Así, una teoría que dice que AGUA significa agua en virtud de que existe la primera ley también se requiere que diga que AGUA significa $\mathrm{H}_{2} \mathrm{O}$ en virtud de que existe la segunda. Razonamientos paralelos muestran que $\mathrm{H}_{2} \mathrm{O}$ significa agua, por ende, que AGUA y $\mathrm{H}_{2} \mathrm{O}$ significan lo mismo.

»[...] De hecho, voy a ser más concesivo aún. Dado mi punto de vista de que el contenido es información, no puedo, tal como vimos, permitirme el lujo de aceptar que el contenido del concepto $\mathrm{H}_{2} \mathrm{O}$ sea diferente del contenido del concepto AGUA. Pero estoy completamente dispuesto a aceptar que son conceptos diferentes. En efecto, estoy suponiendo que las representaciones correferenciales son ipso facto sinónimas y estoy concediendo que, puesto que lo son, la individuación del contenido no puede ser lo único pertinente para la individuación del concepto. [...] Los conceptos se distinguen a partir de dos parámetros (posiblemente ortogonales); a saber, la referencia y el modo de representación (MDR). Así, por ejemplo, el concepto de AGUA es distinto del concepto PERRO según ambos parámetros, pero es distinto del concepto $\mathrm{H}_{2} \mathrm{O}$ sólo respecto del segundo.»

Desde nuestra perspectiva de expertos decimos que entre los MDR AGUA y $\mathrm{H}_{2} \mathrm{O}$ existen muchas diferencias, porque numerosos códigos sintácticos consensuados por expertos se condensan en el formato $« \mathrm{H}_{2} \mathrm{O} »$ (lenguaje formal de la figura 2). Por ejemplo:

- H y O son los símbolos químicos de dos elementos químicos muy reactivos, que se encuentran en estado natural como gases biatómicos o combinados en miles de sustancias compuestas diferentes.

- El subíndice indica atomicidad doble para el hidrógeno y simple para el oxígeno, en una molécula de agua, y es la proporción estequiométrica para una cantidad cualquiera de agua químicamente pura.

- En la molécula de agua, estos elementos químicos no se encuentran en sus estados de carga neutra, sino que han formado enlaces covalentes polarizados, al punto que, de forma indistinguible, uno de ellos está parcialmente ionizado.

- La hibridización del oxígeno es el fundamento teórico que justifica el momento dipolar de la molécula. Éste, a su vez, ocasiona la existencia de fuerzas intermoleculares fuertes entre las moléculas de agua, denominadas puente de hidrógeno. 
Por otra parte, en la mente del experto, el MDR agua, puede remitir a las significaciones de:

- agua de mar;

- agua químicamente pura;

- agua mineral;

- vapor de agua;

- diferentes propiedades para el agua líquida, el hielo y el vapor de agua;

- etcétera.

Por lo tanto, lo que para J. Fodor son modos de representación de un mismo concepto, equivalentes a una dada significación, se revelan -para los que somos expertos en el campo semántico donde se originaron dichos MDRcomo elementos sintácticos codificados que encapsulan gran cantidad de información, con significaciones sumamente complejas. Para ilustrar este punto cabe reflexionar sobre las capacidades de un experto en química para comprender las diferencias y significaciones correctas, o incorrectas, de los ítems de la figura 4; y sobre las dificultades que un no-experto en química -iaunque experto en filosofía!- tendría para igualarlo.

Aprender significaría que un no-experto llegara a manejar lenguajes expertos, comprendiendo sus códigos y formatos sintácticos con los significados que le otorgan los expertos.

\subsection{La construcción semántica: los lenguajes ex- pertos como vínculo u obstáculo de comunicación}

El conocimiento que los expertos guardan en sus mentes no se ve; sólo se hace evidente cuando ellos lo explicitan en algún lenguaje. Las mentes de los expertos tienen gran movilidad representacional, y eso lo demuestran explícitamente al utilizar complementariamente diferentes lenguajes (como los señalados en la figura 2) para expresar sus conocimientos. Los alumnos sólo perciben el aspecto sintáctico del discurso del experto y, a partir de éste, deben construir su propio conocimiento, otorgando a la sintaxis recibida una significación precisa. Es decir, los alumnos deberán reconstruir en sus mentes niveles semánticos similares al que maneja el experto, en cada uno de los lenguajes utilizados. Pero, para lograr esta reconstrucción, sólo disponen de sus propias representaciones mentales de partida, seguramente muy diferentes a las del experto en la disciplina. Esta situación, que se muestra en la figura 5, plantea claramente que, si bien las expresiones sintácticas son las formas explícitas de la comunicación, la interpretación de tales expresiones es idiosincrática, pueden ser entendidas en forma diferente y esto dependerá de cuáles son las representaciones mentales (Bonatti, 2001) de cada sujeto.

Estas dificultades en la comunicación pueden llevar a que, para muchos alumnos, el aprendizaje sustentable de un tema dado sea una tarea imposible.

Para facilitar la reconstrucción apropiada de los significados durante el aprendizaje, es necesario que los alumnos expresen aquello que están construyendo como conocimiento en su mente. Al expresarlo, pueden suscitarse diversos casos, entre los cuales podemos citar dos extremos: expresión sintáctica correcta, pero reconstrucción incorrecta de los respectivos significados; o bien, expresión sintáctica defectuosa o distorsionada, con significación correcta de lo que se está construyendo en sus mentes (Seferián, 2002).

Solicitar a los alumnos que utilicen diferentes lenguajes como medios de expresión ayuda a detectar errores. Frecuentemente ocurre que un aprendizaje aislado o significativo erróneo conduce a expresiones del alumno poco precisas o inválidas, sin que éste se percate de ello. Decimos que éstos son errores inadvertidos sistemáticos. La evaluación sobre qué y cómo se está configurando el aprendizaje en la mente de los alumnos sólo será posible si ellos lo explicitan mientras lo están construyendo. Esta actividad en la clase apunta a lograr coincidencia entre el nivel sintáctico y la reconstrucción semántica apropiada. En la parte 1 de este artículo (Galagovsky, 2004), ejemplificamos este punto analizando el caso de un sujeto que ha aprendido significativa y erróneamente que «en verano hace más calor porque la Tierra está más cerca del Sol».

Las actividades que tienden a mejorar la comunicación entre el docente y los alumnos están requiriendo un mayor tiempo de clase durante el cual los alumnos expliciten los procesos cognitivos incolucrados en la construcción de sus aprendizajes (Jiménez Aleixandre, 1996). Estas actividades definen, desde nuestro punto de vista, qué significa «lograr que los alumnos participen en clase». Propiciar la participación no significa solamente que algún buen alumno conteste a preguntas organizadas mayéuticamente por el docente, sino que la mayoría de los alumnos puedan «mostrar»-hacer explícito- qué están construyendo en su mente; y cómo lo expresan.

Figura 4

Los expertos en química pueden decidir si las siguientes fórmulas tienen significación correcta.

\begin{tabular}{|llll|}
\hline a) $\mathrm{H}_{2} \mathrm{O}$ & b) $\mathrm{H}^{2} \mathrm{O}$ & c) ${ }^{2} \mathrm{HO}$ & d) ${ }_{2} \mathrm{HO}$ \\
$e) \mathrm{OH}_{2}$ & $f){ }^{2} \mathrm{H}_{2} \mathrm{O}$ & $g){ }^{2} \mathrm{H}^{2} \mathrm{O}$ & h) $2 \mathrm{H}_{2} \mathrm{O}$ \\
i) ${ }_{2} \mathrm{H}^{2} \mathrm{O}$ & j) $\mathrm{H}_{2}{ }_{2} \mathrm{O}$ & k) $\mathrm{H}_{2} \mathrm{O}_{2}$ & $l$ l) ${ }^{2} \mathrm{HO}_{2}$ \\
\hline
\end{tabular}


Aspectos sintácticos como vínculo u obstáculo de comunicación entre docente (experto) y alumnos (novatos).

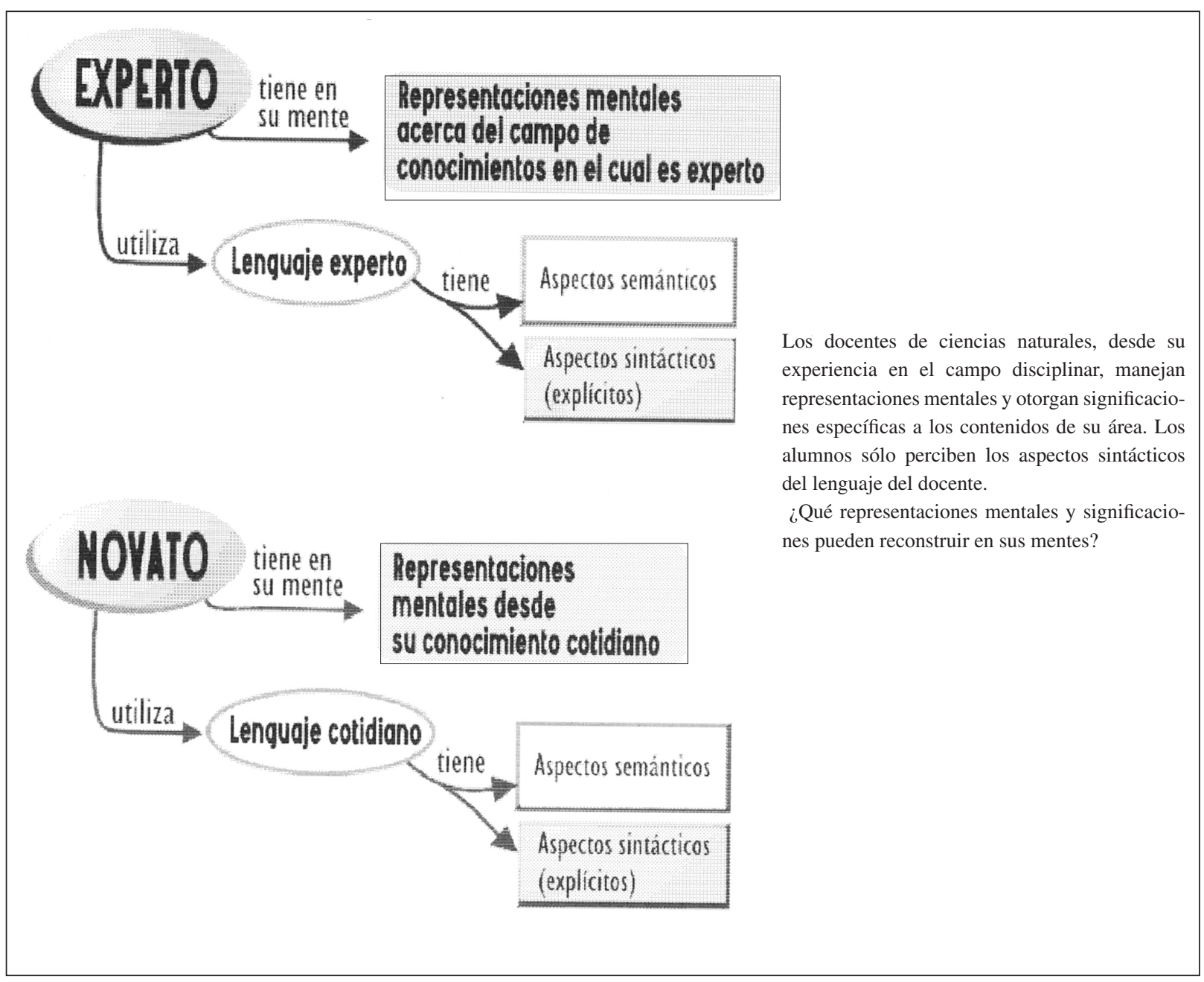

\section{DERIVACIONES DIDÁCTICAS DEL MACCS}

\subsection{Diferencias epistemológicas y didácticas entre indagar ideas previas y traer a la conciencia con- ceptos sostén}

Un frondoso trabajo observando docentes en ejercicio nos ha revelado que existe una tendencia simplista a interpretar la consigna de Ausubel: «Averígüese lo que sabe el alumno y enséñese en consecuencia.» (Novak, 1984). El ritual observado consiste en que el docente, antes de enseñar un tema, efectúa unas preguntas a los alumnos sobre qué saben acerca de dicho tema. Frente a esta consigna, algún alumno enuncia una opinión, algún otro una idea o una declamación memorística de algo relacionado que recuerda haber estudiado antes; el resto de la clase suele ser ajeno a cualquier elaboración mental o profundidad de razonamiento. Una vez cumplido con este ritual de indagar ideas previas, el docente da su beneplácito o su corrección a algo de lo dicho y ya expone el nuevo contenido.

El MACCS cuestiona este ritual, porque las ideas previas pueden ser erróneas o incompletas, y deberían ser modificadas para lograr el tan esperado cambio conceptual. El MACCS reclama que los conceptos sostén deben ser conceptos nexo correctos y estar presentes en la estructura cognitiva del alumno, ya que son los pilares donde se asentará el nuevo conocimiento sustentable.

Esta diferencia marca un trasfondo epistemológico fundamental:

- Los conceptos sostén serán aquéllos sobre los que sí podrá construirse el aprendizaje sustentable.

- Las ideas previas, alternativas o resistentes, se espera 
que sean erróneas. Sobre ellas deberá promoverse un conflicto cognitivo para que se transformen en ideas científicamente correctas y se logre el cambio conceptual (Pozo, 1987, 1997, 1999; Posner et al., 1982; Nussbaum y Novik, 1982; Rodrígez Moneo, 1999).

La consecuencia directa de esta disyuntiva epistemológica es el requerimiento de plantear situaciones didácticas paradigmáticamente muy diferentes. Se expondrá, a continuación, un ejemplo.

\subsubsection{Un ejemplo para el concepto de densidad}

\subsection{1.a. Las preguntas que debería hacerse un docente}

Desde el punto de vista de los paradigmas que se adhieren a una secuencia didáctica del tipo (a) relevar ideas previas, (b) generar conflicto cognitivo, (c) facilitar el cambio conceptual, podemos suponer que un profesor que hubiera querido indagar las ideas previas de sus alumnos sobre el tema «densidad», pudo haber recibido las siguientes respuestas ante la pregunta «¿Qué es densidad?»:

«es algo denso»; o «es cuando un tipo es pesado, fastidioso»; o «es pastoso»; o «es que en la ciudad vive más gente que en el campo»; o «el aceite»; o «es cuando algo es muy pesado y se hunde»; o «es viscosidad»; o «es algo apretado»; o «es lo que tiene un disquete (Ogborn et al., 1998, p. 69)

Este profesor, encantado de oír tantas ideas previas y por la «participación» lograda, se sentiría autorizado para continuar con su clase y ya poder explicar qué es densidad.

Desde el MACCS, interpretamos que en esta situación cada alumno ha traído a su conciencia algunas palabras asociadas a la «palabra-estímulo» densidad, pero que esos conceptos que han aflorado en sus conciencias distan mucho de ser conceptos sostén.

Nuestra postura exige una severa reflexión previa del docente antes de dar una consigna para traer a la conciencia conceptos sostén. La propuesta didáctica derivada del MACCS no consiste en restringir la aparición de estas respuestas idiosincráticas incompletas o erróneas, sino de anticiparlas para elaborar una consigna clara, cuya respuesta sea fácil de relevar. Anticipar estas respuestas, para el tema de densidad, demandaría que el docente reflexionara, por ejemplo, sobre estas cuestiones:

- ¿Cuál es la diferencia entre densidad de un cuerpo y densidad de una población?

El concepto de densidad de una población, es decir, la cantidad de individuos por unidad de superficie -área de biología o sociología-, no requiere que la distribución de la variable sea homogénea. Sin embargo, densidad de un cuerpo implica que el cuerpo sea un sistema homogéneo; para un cuerpo heterogéneo hablaríamos de peso específico.
Desde el aspecto procedimental, en ciencias sociales, el cálculo de una densidad de población puede traer aparejado problemas de muestreo, mientras que, en ciencias naturales, tiene que ver con mantener fijas variables tales como la temperatura, la concentración (si fuera la densidad de una solución) y la sensibilidad de los instrumentos de medición (de masa y volumen).

- En ciencias naturales, ¿cuál es la diferencia entre densidad de un cuerpo y densidad de un fluido? La densidad referida a un fluido requiere fijar la temperatura del sistema, pero, para la densidad de un cuerpo sólido, en general no se requiere este tipo de dato.

- La densidad (en física y química) es una proporción entre las magnitudes masa y volumen, ¿cuál es la diferencia, entonces, entre los conceptos de densidad y concentración?

- La densidad de información es un dato cuantitativo o cualitativo? ¿En qué circunstancias?

- ¿Cuál es la diferencia entre densidad y viscosidad?¿Por qué parecen sinónimos para el lego?

La consigna inicial, entonces, podría consistir en armar situaciones problemáticas simples, concretas y entendibles para los alumnos. Sin pretender ser exhaustivos en el presente trabajo, sugerimos una consigna como se muestra en la figura 6.

Las respuestas de los alumnos, al expresarse en forma concisa podrán ser fácilmente relevadas y discutidas en el pequeño grupo y luego ser comunicadas en la puesta en común. Al efecto de continuar con el ejemplo, podríamos suponer tres tipos de respuestas «erróneas», para efectuar un posterior análisis desde el MACCS. Estas respuestas erróneas podrían ser:

a) En el campo A hay mayor densidad de vacas porque están las 10 vacas bien juntas, apretadas. En los otros campos están más separadas.

b) En el campo B hay mayor densidad de vacas porque hay dos vacas más que en $\mathrm{A}$ y 7 más que en $\mathrm{C}$.

c) En el campo C hay mayor densidad de vacas porque hay menos lugar.

Estas posibles respuestas erróneas remiten a rasgos perceptibles de los esquemas de la figura 6. Las respuestas apuntan a magnitudes absolutas, en tanto que densidad de población es una magnitud relativa.

¿Qué conceptos sostienen en las mentes de los alumnos cada una de estas respuestas?

La primera respuesta remitiría a que mayor densidad es algo que significa estar «más junto, más apretado». Es un término aparentemente relativo, pero expresado de tal forma que sólo tiene en cuenta el valor absoluto de la cantidad de «ese algo». 
Consigna para hacer llegar a la conciencia conceptos nexo sobre el concepto de densidad.

Los cuadrados A, B y C representan campos y los óvalos negros son vacas. ¿En qué campo hay mayor densidad de vacas? ¿Por qué?

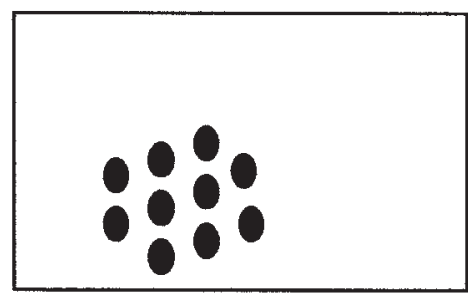

A

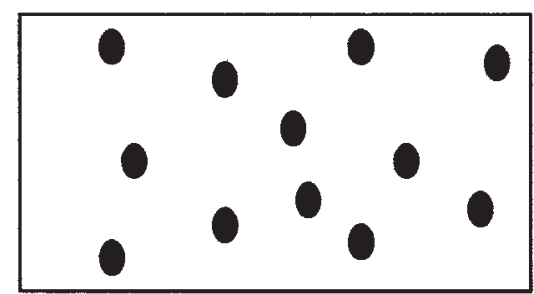

B

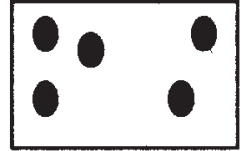

C

Superficies: campo A, 15 ha; campo B, 18 ha; campo C, 7,5 ha

La segunda respuesta remitiría a la significación de que «mayor densidad» es cuando hay mucho de algo; también remitiría a la cantidad como valor absoluto.

La tercera respuesta referiría el concepto de mayor densidad a «algo que se encuentra en un espacio reducido»; estaría relacionado con la significación de la primera respuesta, pero teniendo en cuenta el espacio y no los objetos que están en él.

Vemos cómo, en este ejemplo, una pregunta bien planteada, apunta a respuestas estructuradas que evidencian los conceptos nexo de estas significaciones alternativas erróneas que dan los alumnos a la palabra densidad. Ahora bien, el docente sabe que sobre estos conceptos nexo no se puede construir el concepto de densidad desde su significación en ciencias naturales. ¿Con qué tipo de acciones puede continuar?

\subsection{1.b. Las acciones didácticas que tradicionalmente plan- tean los docentes}

Nuevamente, sin pretender con este ejemplo cubrir todas las posibilidades, propondremos y analizaremos dos tipos extremos de acciones que el docente podría plantearse para trabajar con los alumnos.

Desde los paradigmas que señalan la necesidad de indagar ideas previas para, finalmente, lograr un cambio conceptual, surge que el docente puede continuar la clase comunicando que todas las respuestas son incorrectas o, al menos, incompletas. A continuación, daría la información correcta sobre cómo se define la magnitud densidad de población en términos de cociente entre la cantidad de objetos y la superficie en que se encuentran. Luego, plantea su homologación a la definición de densidad para las ciencias naturales.
Esta opción comporta poco tiempo y su estrategia puede resumirse de la siguiente manera:

a) Los alumnos exponen sus respuestas (sus ideas previas).

b) Se consideran indagadas las ideas previas, que se hicieron explícitas.

c) Se brinda la «información correcta» con la intención de generar un conflicto cognitivo en los alumnos.

Esta estrategia puede expresarse gráficamente, según los códigos gráficos del MACCS, como se muestra en la figura 7. En 7a, el docente da una consigna para que un alumno exprese sus ideas previas; en $7 \mathrm{~b}$, el alumno procesa, en su memoria de corto plazo, la pregunta, busca asociaciones en su memoria de largo plazo; en $7 \mathrm{c}$, el alumno organiza en su mente (memoria de trabajo) su respuesta, a partir de sus conceptos nexo idiosincráticos y expresa su respuesta en algún lenguaje. En la clase, podemos suponer que varios alumnos exponen sus respuestas. En la figura 7d, el docente da la información correcta a su pregunta inicial, es decir, enseña el tema. El docente tiene en su mente los conceptos sostén apropiados que otorgan significado a la expresión sintáctica de su respuesta. Esta acción del docente puede tener la intención de generar un conflicto cognitivo en sus alumnos (en la sección 3.2 veremos por qué desde el MACCS esto se cuestiona). En las figuras $7 \mathrm{c}$ y $7 \mathrm{~d}$ el código gráfico utilizado para los nodos de la información son distintos; con esto pretendemos representar que el alumno explicita su respuesta en un lenguaje poco científico, utilizando sus propios códigos y formatos sintácticos, muy diferentes de aquéllos utilizados por el lenguaje de la ciencia erudita empleado por el docente. 
Figura 7

El conflicto cognitivo.
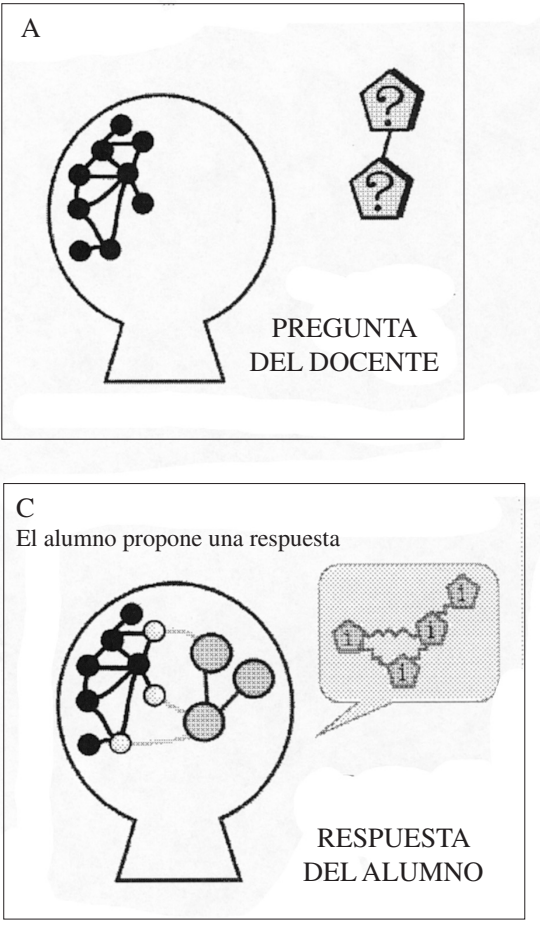

\section{D}

El docente expone la respuesta correcta, validada por la ciencia

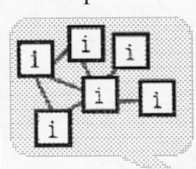

RESPUESTA CORRECTA
B
El alumno procesa la pregunta en su MCP

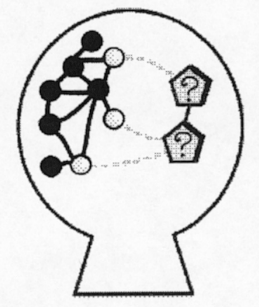

E

¿De quién es el conflicto cognitivo planteado entre las dos informaciones contradictorias?

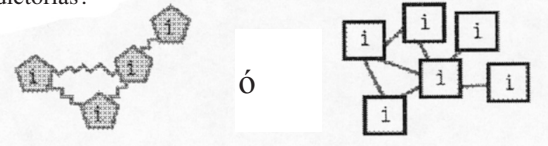

Estrategia docente en la cual: a) el docente da una consigna para que un alumno exprese sus ideas previas; $b$ ) el alumnos procesa en su memoria de corto plazo la pregunta y busca asociaciones en su memoria de largo plazo; c) el alumno organiza en su mente (memoria de trabajo) su respuesta a partir de sus conceptos sostén idiosincráticos; $d$ ) el docente da la información correcta a su pregunta inicial.

\subsection{1.c. Las acciones didácticas que deberían planifi- carse desde el MACCS}

Desde el MACCS se sugiere que el docente facilite que los alumnos tomen conciencia sobre las características de los conceptos nexo que han originado cada respuesta. El consenso debe llevarlos a construir en sus mentes el concepto apropiado de densidad de población, como una magnitud relativa, originada a partir de dos magnitudes absolutas (la cantidad de objetos y la superficie en la que se encuentran). La estrategia de este docente puede resumirse de la siguiente forma:

a) Los alumnos exponen sus respuestas (sus ideas previas sostenidas por conceptos nexo equivocados o incompletos).

b) Se propicia la identificación consciente y la caracterización de los conceptos nexo involucrados en dichas res- puestas, partiendo del hecho real que para cada alumno su respuesta es correcta y tiene significado.

Los alumnos construyen el concepto apropiado de densidad de población a partir de que comprendan que sus percepciones los han llevado a explicitar una magnitud absoluta en vez de una relativa, como lo es la densidad de población. Adviértase que se han valorado las respuestas de los alumnos como base, como sostén imprescindible, para la «re-construcción» de un nuevo concepto sostén, más complejo y sobre el que sí se podrá seguir construyendo aprendizaje sustentable.

Para que los alumnos construyan el significado de densidad, desde ciencias naturales, el docente deberá planear nuevas preguntas, sabiendo que ya existe en las mentes de los alumnos el concepto de densidad de población, como un cociente entre dos magnitudes absolutas (cantidad de algo y superficie). Proponemos 
que éste sea el concepto sostén sobre el que podrá construirse -homologando a tres dimensiones- el concepto de densidad de un cuerpo, como el cociente entre su masa y su volumen.

\subsection{Redefinición conceptual y operativa de los pro- pósitos didácticos de generar conflicto cognitivo y lograr un cambio conceptual}

\subsubsection{Breve reseña de los antecedentes existentes}

En los últimos años, en el contexto de las investigaciones sobre concepciones espontáneas erróneas en los alumnos con respecto a los fenómenos científicos, han surgido numerosas teorías de aprendizaje que conciben a éste como un proceso de cambio conceptual. El fundamento de estos enfoques supone que el progreso de las ideas científicas en el conocimiento de quien aprende implica reestructuraciones, ya sean éstas fuertes o débiles (Carey, 1985). Las teorías sobre el cambio conceptual están basadas, fundamentalmente, en estudios epistemológicos acerca del progreso de las ideas científicas analizadas en diferentes campos disciplinares como el de las ciencias fácticas (Kuhn, 1962; Lakatos, 1978), el de la psicología del desarrollo (Piaget y García, 1983; Moreno, 1988, Pozo, 1987) o el de la psicología del aprendizaje (Carey, 1985; Chi, Glaser y Farr, 1988).

A partir de estos enfoques teóricos se han propuesto numerosos abordajes instruccionales que tratan de identificar estrategias didácticas que fomentan el cambio conceptual en los alumnos (Hewson y Hewson, 1984; Nussbaum y Novik, 1982; Osborne y Freyberg, 1985; Osborne y Wittrock, 1983; Cosgrove y Osborne, 1985; Posner et al., 1982; Pozo, 1997). La idea principal de estos abordajes es que el aprendizaje de conceptos científicos debe partir de los conceptos naturales que ya posee el alumno: la «ciencia intuitiva» con la que el alumno acude al aula.

Pozo (1997) dice que enseñar ciencias no consistiría en proporcionar conceptos a los alumnos sino en cambiar los que poseen; no bastaría con exponer al alumno a un modelo explicativo mejor, hay que hacerle ver que es mejor. «Para que el alumno pueda comprender la superioridad de la nueva teoría es preciso enfrentarlo a situaciones conflictivas que supongan un reto para sus ideas. De esta forma, el conflicto cognitivo es muy importante en el avance conceptual del alumno, aunque en ningún caso debe considerarse condición suficiente para el cambio conceptual».

Finalmente, se acepta que la toma de conciencia por parte del alumno sobre sus teorías iniciales, muchas veces implícitas, es un paso indispensable para el cambio conceptual.

Una revisión exhaustiva de los modelos de aprendizaje basados en el cambio conceptual y de las características de los conflictos cognitivos asociados a cada modelo pueden verse en Pozo (1997) y Rodríguez Moneo (1999).

\subsubsection{Una necesaria discriminación entre situaciones de aula tendientes a generar conflictos cognitivos}

Cabría reflexionar sobre la existencia de diferentes situaciones tendientes a generar un conflicto cognitivo. Estas pueden surgir a partir de:

a) la mostración de una realidad experimental cuyo resultado empírico no coincide con el predicho por los alumnos desde sus teorías implícitas;

b) la significación diferencial de un término conocido, con una utilización en otro contexto teórico;

c) la utilización de terminología cuya significación desconocen los alumnos;

d) la presentación de información científica correcta que contradice o refuta y descalifica las ideas previas expresadas por los alumnos.

En el primer caso, está claro que los alumnos no podrán dar fácilmente las explicaciones científicas correctas, porque eso supondría un aprendizaje por descubrimiento y no podemos esperar que, en la mente del alumno, ocurran en breve lapso, los procesos cognitivos que demandaron decenas de años o siglos de evolución del pensamiento de la comunidad científica (Barrón Ruiz, 1993). Sin embargo, no cabe duda de que este tipo de actividades promueve motivación cuando la predicción ha sido verdaderamente efectuada por los alumnos. Esta motivación no se logra, en la misma medida, cuando la experiencia práctica consiste sólo en confirmar lo ya estudiado teóricamente y predicho por la ciencia -o por la guía de trabajos prácticos.

En el segundo caso, generalmente, el alumno no es consciente de esta múltiple significación, lo cual conduce a aprendizajes significativos erróneos al utilizar conceptos nexo equivocados.

El tercer caso genera situaciones angustiantes en el alumno, pues no puede procesar la información que está recibiendo, al no reconocer en su estructura cognitiva conceptos nexo para dicho procesamiento.

El cuarto caso remite a la significación más frecuente dada por los docentes a la acción de generar un conflicto cognitivo conciente en los alumnos, y coincide con la planificación analizada en el ítem 3.1.1.b.

Desde el MACCS, se espera otro tipo de acciones didácticas (sección 3.1.1.c), dado que hemos detectado lo que consideramos una falla epistemológica en la concepción de cómo se construye un conflicto cognitivo en la mente de los alumnos, según se detalla continuación.

\subsubsection{El MACCS demanda la existencia de conceptos sos- tén para poder procesar un conflicto cognitivo}

El concepto de conflicto cognitivo es entendido, generalmente, como una incoherencia de la que se toma conciencia; es decir, sería una percepción consciente 
de una contradicción entre dos informaciones. Hemos mostrado en la figura 7 (a, b, c, d) las secuencia de acciones didácticas frecuentemente usadas para generar un conflicto cognitivo en los alumnos (sección 3.1.1.b). La figura 7e nos plantea una pregunta crucial: ¿quién puede procesar la contradicción entre ambas informaciones?

\section{Si sabemos que:}

a) la respuesta del docente proviene de trasposiciones didácticas hechas sobre el contenido científico erudito; tiene terminología precisa; utiliza lenguajes complementarios con códigos y formatos sintácticos consensuados por los expertos;

b) el docente tiene en su estructura cognitiva los conceptos sostén apropiados para sostener comprensivamente su propio discurso científico;

c) el docente tiene la capacidad cognitiva de reconocer la respuesta del alumno como total o parcialmente errónea; a veces, puede comprender o interpretar qué quiso decir el alumno y hasta puede darse cuenta de las fallas conceptuales de esta respuesta. Es decir, el docente tendría en su estructura cognitiva los conceptos sostén apropiados para comprender tanto su respuesta como, también, las fallas manifestadas en la respuesta del alumno;

d) el alumno que ha elaborado una respuesta, la comprende y le da significado si, con gran esfuerzo de búsqueda y replanteos en su estructura cognitiva, pudo organizarla en su mente y expresarla. Sin embargo, este alumno todavía no ha constituido en su mente los conceptos sostén apropiados para procesar la respuesta del docente (si los tuviera, habría logrado una aprendizaje sustentable al poder procesar esa información científica). Por lo tanto, percibe la respuesta del docente como totalmente incomprensible.

Entonces, tal como se muestra en la figura 8, es en exclusiva el docente quien percibe conscientemente el conflicto cognitivo; es decir, quien comprende la contradicción entre las dos informaciones (la respuesta del alumno y su propia explicación), porque tendría en su mente los conceptos sostén para procesarlas a ambas ${ }^{3}$.

Frente a esta realidad, en la cual el alumno recibe la información científica correcta (Ítems a, b, c de la sección 3.1.1.b), entendemos que los conflictos de los alumnos suelen ser los siguientes:

- ¿Para qué el profesor me pregunta si yo no sabía lo que había que responder?

- ¡Jamás se me iba a ocurrir esta respuesta! Si ni siquiera entiendo lo que quiere decir.

Y su aprendizaje puede ser:

«La próxima vez no me arriesgo a hablar porque quedo como un tonto ante el profesor y ante mis compañeros, dando una respuesta equivocada.»

El dueño de un conflicto cognitivo consciente es el individuo que toma conciencia de la contradicción entre dos informaciones.

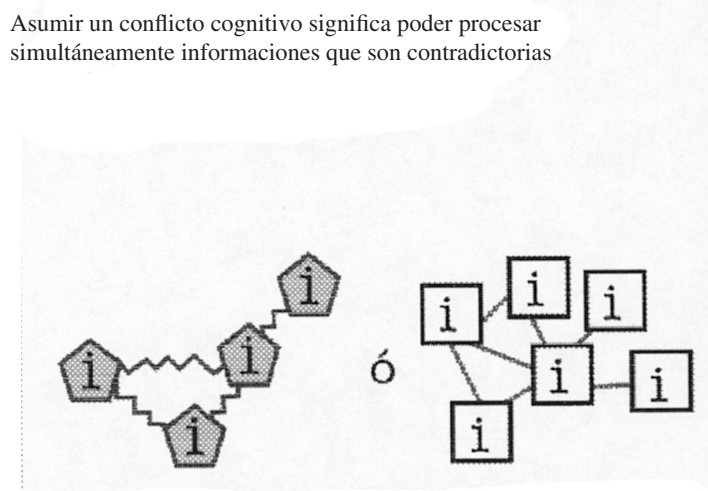

INFORMACIONES MUTUAMENTE EXCLUYENTES

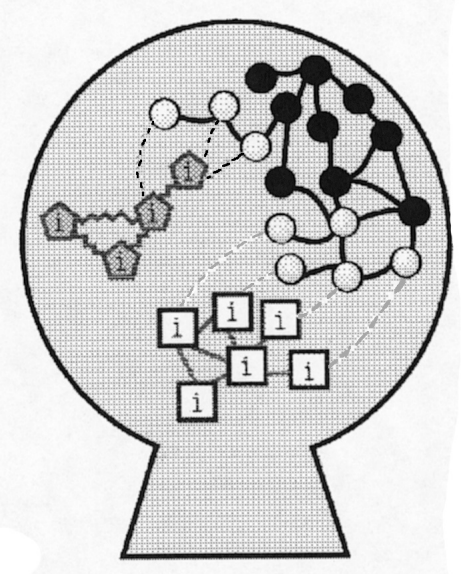




\subsection{Cuestionamiento del enfoque tradicional sobre el cambio conceptual}

Son numerosas las investigaciones que apuntan a determinar y caracterizar las destrezas cognitivas que distinguen a los expertos de los novatos; tales como las de Larkin y otros (1980), Simon y Simon (1978), Larkin (1980, 1983), Chase y Simon (1973), Hart (1986), Chi, Glaser y Rees (1982), De Kleer (1977), entre otros. Muchas de estas investigaciones consisten en descripciones y categorizaciones de procesos cognitivos de los expertos y su comparación con la de los novatos, y apuntan a la resolución de problemas diferentes, de tal manera que los resultados de tales investigaciones no son estrictamente comparables.

El término cambio conceptual en el ámbito educativo está ligado, fundamentalmente, al esperado cambio de las ideas intuitivas de los alumnos por las ideas científicamente correctas de los expertos (Pozo, 1997).

El MACCS extiende su mirada crítica sobre este punto y establece que una cosa es la investigación sobre los procedimientos cognitivos diferenciales entre expertos y novatos frente a un problema dado y otra es suponer que, porque el docente experto brinde información científicamente correcta, se va a lograr que los alumnos, fácilmente, tomen conciencia de sus conflictos conceptuales o procedimentales y los superen.

El MACCS encierra una potencialidad respecto a la resignificación necesaria del concepto de cambio conceptual en contextos de enseñanza que deberá ser, aún, analizada y profundizada en sucesivos trabajos. Sin embargo, por lo expresado hasta aquí, puede adelantarse que este cambio conceptual estará ligado a los cambios en las representaciones mentales de los alumnos, a partir de:

a) la identificación consciente sobre los conceptos nexo que les dan significaciones a sus representaciones mentales incompletas o erróneas;

b) la posibilidad de motivarse frente a conflictos cognitivos procesables en su memoria de trabajo para lograr la revisión consciente de dichos conceptos nexo;

c) la resignificación consciente de los conceptos nexo incompletos o erróneos y la consecuente construcción de los conceptos sostén apropiados;

d) la construcción consciente de aprendizajes sustentables sobre conceptos sostén y su análisis metacognitivo con respecto a las representaciones mentales de partida.

Desde el MACCS se percibe el cambio conceptual como un progresivo enriquecimiento tanto en destrezas cognitivas como en conocimientos, por vía de aprendizajes sustentables. En esta progresión de crecimiento cognitivo, el sujeto podrá atravesar situaciones de conflictos cognitivos conscientes; lo que significa que el sujeto tomará conciencia y podrá procesar en su memoria de trabajo informaciones contradictorias, hasta lograr resolverlas. Estos conflictos cognitivos aparecen, fundamentalmente, durante el trabajo consciente de identificación, selección, resignificación reconstrucción de los conceptos nexo iniciales en su camino a convertirse en conceptos sostén.

El rol docente es el de proponer situaciones didácticas que permitan a los alumnos hacer llegar a la conciencia potenciales conceptos nexo y dar el tiempo y las consignas necesarias para que ellos trabajen sobre dichos conceptos hasta encontrar los conceptos sostén apropiados ${ }^{4}$.

El MACCS entiende el cambio conceptual como una continuidad en la construcción del conocimiento más que como una ruptura o una discontinuidad epistemológica. El cambio conceptual ocurriría cuando los alumnos logren armar redes apropiadas de conceptos sostén y sean capaces de procesar información científica y convertirla en conocimiento sustentable. En realidad, el cambio como ruptura epistemológica se daría, eventualmente, en la modificación consciente de las significaciones originales de los conceptos nexo o sus funciones dentro de las representaciones mentales iniciales.

\subsection{Utilización crítica del tiempo de clase: dar in- formación es más rápido que apuntar a un apren- dizaje sustentable}

Hay un punto muy importante a tener en cuenta cuando se quiera construir un conocimiento sustentable: cómo se reparte el tiempo de clase. Nuestras sugerencias son:

- Se debe otorgar más tiempo a los procesos cognitivos de los alumnos, particularmente a aquéllos que implican traer a sus conciencias conceptos nexo, explicitarlos y discutirlos con sus pares y el docente hasta llegar a consensos de significación. Éste no es tiempo perdido; es tiempo invertido en mejorar la calidad del aprendizaje (Galagovsky, 1993).

- Se debe alterar el orden tradicional de actividades. En lugar de que el docente aporte primero la información «correcta», luego presente un ejercicio tipo y, finalmente, los alumnos repitan la información recibida, sugerimos que los alumnos deberían trabajar primero sobre sus propias mentes, a partir de una consigna apropiada, transformando sus conceptos nexo y representaciones mentales originales en conceptos sostén, que les permitan procesar la información científica y transformarla en conocimiento sustentable.

- Se debe reconsiderar el tiempo de trabajo en clase en el sentido de lograr que sean los alumnos los que trabajen -pensando, expresándose, comparando aspectos sintácticos y significaciones- en lugar de tener al docente hablando y explicando y a los alumnos sólo copiando. La idea es que los alumnos estén activos durante la clase al argumentar con sus pares, producir y revisar sus propios conocimientos, tomar conciencia de sus conceptos nexo erróneos o incompletos y de sus conceptos sostén apropiados, y de los lenguajes que utiliza para expresarlos. Entendemos la participación de los alumnos como una 
construcción, un enriquecimiento, una resignificación y revisión metacognitiva permanente de los contenidos de sus respectivas estructuras cognitivas.

No es imprescindible que estas sugerencias sean aplicadas a la totalidad de las clases; pero sí creemos que: a mayor cantidad de conceptos a enseñar en programas extensísimos, más estaremos forzando al docente para que dedique su tiempo de clase a explicar, es decir, a hablar frente a un grupo de alumnos pasivos. Esto significa -y está validado por las mediciones de fracaso escolar en ciencias naturales- que sólo una pequeña proporción de alumnos motivados y capacitados por sí mismos en las destrezas cognitivas requeridas logran procesar la información del profesor mediante un aprendizaje sustentable. Lamentablemente, como señala Gabel (2002), la gran mayoría de los alumnos aprenderá datos aislados con gran esfuerzo memorístico.

Pensemos en la siguiente analogía: ¿qué eficiencia de producción lograría un agricultor que tirara sus semillas sin preparar antes el campo? Si los contenidos a enseñar son análogos a las semillas, las mentes de los alumnos al campo, y la enseñanza -tal como la agricultura- una forma de cultura, saque el lector sus propias conclusiones (Galagovsky, 1993a).

\section{CONCLUSIONES}

Los docentes e investigadores que estamos inmersos en las problemáticas de la didáctica de las ciencias, sabemos que, para enseñar ciencia, no basta con que el profesor sepa el tema y lo exponga con claridad, particularmente cuando la mayoría de los alumnos no van a seguir carreras científicas.

Este artículo, en sus partes 1 y 2, presenta un modelo de aprendizaje simple, el modelo de aprendizaje cognitivo consciente sustentable (MACCS) y sus derivaciones comunicacionales y didácticas. Se trata de un modelo porque se basa en simplificaciones de procesos cognitivos sumamente complejos y permite predecir situaciones tendientes a mejorar la comunicación entre el docente y sus alumnos durante el acto comunicacional de la enseñanza así como a interpretar fallas en el mismo.

Nuestra ambición es llegar a describir algunos procesos mentales preparatorios de la mente de los alumnos para que ésta esté óptimamente preparada para recibir la información científica. Esta información erudita debe ser cuidadosamente revisada en función del MACCS para planificar la trasposición didáctica que se plasmará en las actividades a desarrollarse en clase.

Nuestro MACCS descarta la posibilidad que sean los alumnos quienes puedan descubrir por sí mismos la trama conceptual de la información erudita que deberán aprender y considera que las diferencias en las habilidades cognitivas de expertos y novatos deben reconsiderarse y redefinirse a la luz de los objetivos de aprendizaje que se pretenden en la escuela.

En este artículo hemos mostrado que el MACCS tiene elementos teóricos que, en forma verbal y gráfica, hacen reconsiderar y redimensionar técnicas de enseñanza frecuentemente utilizadas, transformándolas desde un cambio en la visión epistemológica que involucra los procesos de construcción del aprendizaje. Las principales derivaciones comunicacionales y didácticas del MACCS, comparadas con las acciones prescriptas por otros paradigmas de enseñanza, son:

- Generar situaciones didácticas para facilitar la toma de conciencia sobre los conceptos nexo que los alumnos pretenden utilizar para procesar la información científica hasta que ellos encuentren los conceptos sostén apropiados, en lugar de indagar ideas espontáneas de los alumnos y luego señalarlas como erróneas y, simplemente, dar la información científica correcta.

- Facilitar las argumentaciones entre los alumnos, a favor o en contra de la utilización de los potenciales conceptos nexo explicitados por ellos, y sobre sus formas idiosincráticas de procesar la información científica, en lugar de pretender generar un conflicto cognitivo presentando la información científica final contradictoria a las ideas previas de los alumnos.

- Significar al cambio conceptual como un enriquecimiento sustentable, como una construcción continua de la estructura cognitiva de cada alumno, tanto en estrategias como en conocimientos, a partir de sus posibilidades idiosincráticas iniciales - posibilidades desconocidas por el docente- en lugar de suponer que el cambio conceptual ocurrirá cuando el alumno descarte sus ideas y significaciones previas y las cambie por el solo hecho de recibir la información científica correcta que le presenta el docente.

Si bien estas sugerencias de modificar prácticas docentes se establecen en muchas propuestas didácticas concretas y también se proponen como recomendaciones en las conclusiones de numerosas investigaciones en enseñanza y apredizaje específicas para un contenido, esta vez surgen a partir de un modelo teórico de aprendizaje. Esto representa un cambio en la concepción misma sobre qué es la didáctica, ya que, en lugar de llegar a tales conclusiones inductivamente -desde las experiencias y vivencias de aula-, éstas se derivan, deductivamente, de una reflexión de segundo orden sobre la realidad -un modelo- y, por lo tanto, tienen un nivel de abstracción que las hace aplicables a diferentes situaciones y disciplinas. 


\section{NOTAS}

${ }^{1} \mathrm{Si}$ los aprendizajes significativos erróneos llegan a consolidarse como conocimiento, se constituyen en ideas previas, alternativas o intuitivas -misconceptions - frente a posteriores instancias de aprendizaje. Emergen como verdaderos obstáculos epistemológicos, ya que son persistentes, aún después de haberse recibido y aparentemente aceptado la enseñanza correcta del tema en cuestión. Estas ideas se distinguen de los prerrequisitos, que serían conocimientos correctos que deben tener presentes los alumnos para poder seguir aprendiendo un tema.

${ }^{2}$ Convención tipográfica del libro de Fodor: los nombres de los conceptos están escritos en mayúsculas, los nombres de los valores semánticos de las palabras y los conceptos están escritos en cursiva.

${ }^{3}$ A veces, sucede que el docente reconoce como errónea la respuesta del alumno, pero no puede siquiera imaginarse qué conceptos nexo erróneos utilizó dicho alumno para elaborar tal respuesta; o bien supone cuáles han sido esos conceptos nexo erróneos. La forma de allanar esta brecha en la comunicación es plantear actividades que permitan a los alumnos explicitar sus conceptos nexo.
${ }^{4}$ Cabría preguntarse si los conceptos sostén que dan anclaje a un dado conocimiento sustentable en la mente del docente son idénticos o diferentes a los de un alumno que hubiera logrado un aprendizaje sustentable. Creemos que este punto no tiene una respuesta única ni fija, ya que el docente, conocedor de las posibilidades cognitivas de su grupo de alumnos, siempre parcializa la cantidad y profundidad erudita de los conceptos que quiere enseñar. Es obvio que en toda trasposición didáctica hay una simplificación de los contenidos y, por lo tanto, una diferenciación entre los conceptos sostén requeridos para dicho nivel de simplificación de los contenidos. También es obvio que la riqueza de la estructura cognitiva del docente le permite utilizar una gran cantidad de conceptos sostén para significar a cada tema, imposible de ser igualada por el alumno, que recién está construyendo el contenido de dicho campo semántico.

Una cuestión sí es contundente: el docente puede figurarse cuáles son los posibles conceptos sostén accesibles en la mente de sus alumnos, mientras que los alumnos no podrían jamás figurarse cuáles son los conceptos sostén del docente, ya que, para ello, deberían tener una experiencia similar a la suya en el campo científico involucrado.

\section{REFERENCIAS BIBLIOGRÁFICAS}

BARRÓN RUIZ, A. (1993). Aprendizaje por descubrimiento: principios y aplicaciones inadecuadas. Enseñanza de las Ciencias, 11(1), pp. 3-11.

BONATTI, L. L. (2001). Thought, en Lenguage, Brain and Cognitive Development. Essays in honor of Jacques Mehler. Dupoux, E. (ed.). Cambridge, MA: MIT Press.

CAREY, S. (1985). Conceptual change in childhood. Cambridge, MA: MIT Press.

COSGROVE, M. y OSBORNE, R. (1985). Lesson frameworks for changing childrens ideas, en Osborne y Fryberg (eds.). Learning in Science. Hong Kong: Heinemann.

CHASE, W.G. y SIMON, H.A. (1973). Perception in chess. Cognitive Psycology, 4, pp. 55-81.

CHI, M.T.H., GLASER, R. y FARR, M. (eds.) (1988). The nature of expertise. Nueva York: Hillsdale, NJ, Erlbaum.

CHI, M.T.H., GLASER, R. y REES, E. (1982). Expertise in solving problem, en Sternberg (ed.). Advances in the psychology of human intelligence, 1. Nueva York: Erlbaum, Hillsdale.

DE KLEER, J. (1977). Multiple representation of knowledge in a mechanics problem solver. Proceedings of the $5^{\text {th }}$ International Joint Conference on Artificial Intelligence, MA: MIT Press. Cambridge.

FODOR, J.A. (2000). The mind doesn't work that way: The scope and limits od computational psychology. Cambridge, MA: MIT Press.

FODOR, J.A. (1998). Conceptos. Donde la ciencia cognitiva se equivocó. Madrid: Gedisa.

GABEL, D. (2002). Foreword, en Gilbert, J.K., De Jong, O., Justi, R., Treagust, D.F., Van Driel, J.H. (eds.). Chemical Education: Towards Research-based Practice. Dordrecht: Kluwer Academic Publishers.

GALAGOVSKY, L.R. (1993a). Hacia un mejor aprendizaje. Claves en psicoanálisis y medicina. Buenos Aires: Catálogos. 
GALAGOVSKY, L.R. (1993b). Hacia un nuevo rol docente. Una propuesta diferente para el trabajo en el aula. Buenos Aires: Troquel.

GALAGOVSKY, L.R. (1999). Redes conceptuales: memoria, comunicación y aprendizaje. 2a. ed. Buenos Aires: Lugar.

GALAGOVSKY, L.R. (2004) Del aprendizaje significativo al aprendizaje sustentable. Parte 1: el modelo teórico. Enseñanza de las Ciencias, 22(2), pp. 229-240.

GALAGOVSKY, L.R., RODRÍGUEZ, M.A., STAMATI, N. y MORALES, L.F. (2003). Representaciones mentales, lenguajes y códigos en la enseñanza de ciencias naturales. Un ejemplo para el aprendizaje del concepto de reacción química a partir del concepto de mezcla. Enseñanza de las Ciencias, 21 (1), pp. 107-121.

HART, A. (1986). Knowledge acquisition for expert systems. Londres: Kogan Page.

HEWSON, P.W. y HEWSON M.G.A. (1984). The role of conceptual conflict in conceptual change and design of science instruction. Instructional Sciencia, 13, pp. 1-13. Netherlands: Elsevier.

JIMÉNEZ ALEIXANDER, M.P. (1996). Dubidar para aprender. Vigo: Biblioteca Didáctica.

KUHN, T.S. (1971). La estructura de las revoluciones científicas. México: Fondo de Cultura Económica.

LAKATOS, I. (1983). La metodología de los programas de investigación científica. Madrid: Alianza.

LARKIN, J.H. (1980). Teaching problem solving in physics: the psychological laboratory and the practical classroom, en Tuma y Reif (eds.). Problem solving and education. Nueva York: Erlbaum, Hillsdale.

LARKIN, J.H. (1983). The role of problem representation in physics, en Gentner y Stevens (eds.). Mental Models. Nueva York: Erlbaum, Hillsdale.

LARKIN, J.H., McDERMOTT, J., SIMON, D.P. y SIMON H.A. (1980). Expert and novice performance in solving physics problems. Science, 208, pp. 1335-1342.

RODRÍGUEZ MONEO, M. (1999). Conocimiento previo y cambio conceptual. Buenos Aires: Aique.

MORENO, M. (1988). Imaginación y ciencia, en Moreno, M. (coord.). Ciencia, aprendizaje y comunicación. Barcelona: Laia.
NOVAK, J.D. (1984). Teoría y práctica de la educación. Madrid: Alianza.

NOVAK, J.D. (1999). Conocimiento y aprendizaje. los mapas conceptuales como herramientas facilitadoras para escuelas y empresas. Madrid: Alianza.

NUSSBAUM, J. y NOVICK, S. (1982). Alternative frameworks, conceptual conflict and accomodation: Towards a principled teaching strategy. Instructional Science, 11, pp. 183-200.

OGBORN, J., KRESS, G., MARTINS, I. y McGILLICUDDY, K. (1998). Formas de explicar. La enseñanza de las ciencias en secundaria. Madrid: Aula XXI, Santillana.

OSBORNE, R.J. y FREYBERG, P. (eds.) (1985). Learning in science: The implications of childrens' science. Nueva Zelanda: Heinemann Educational.

OSBORNE, R.J. y WITTROCK, M.C. (1983). Learning science a generative process. Sciencie Education, 67, pp. 489-508.

PIAGET, J. y GARCÍA, R. (1983). Psicogénesis e historia de las ciencias. México: Siglo XXI.

POSNER, G.J., STRIKE, K.A., HEWSON, P.W. y GERTZOG, W.A. (1982). Accomodation of a scientific conception: toward a theory of conceptual change. Science Education, 66(2), pp. 211-227.

POZO, J.I. (1987). La historia se repite: las concepciones espontáneas sobre el movimiento y la gravedad. Infancia y Aprendizaje, 38, pp. 69-87.

POZO, J.I. (1999). Más allá del cambio conceptual: el aprendizaje de la ciencia como cambio representacional. Enseñanza de las Ciencias, 17(3), pp. 513-520.

POZO, J.I. (1997). Teorías cognitivas del aprendizaje. Madrid: Morata.

SEFERIAN, A. (2002). "Aplicación de un análogo concreto en situacion de modelo didáctico analógico (MDA) para el estudio de la teoría cinético-molecular de los gases». Tesis de licenciatura en enseñanza de las ciencias. Universidad de General San Martín. Buenos Aires.

SIMON, D.P. y SIMON, H.A. (1978). Individual differences in solving physics problems, en Siegler (ed.), Children's thinking: what develops? Nueva York: Erlbaum, Hillsdale. 